\title{
Periodontitis en la diabetes tipo 2 y la copaiba como coadyuvante del tratamiento: Reporte de casos
}

\section{Periodontitis in diabetes and copaiba as a coadjuvant of the treatment: Case reports}

D. Ramos-Perfecto*, L. Maita Véliz** , L. Mariano Maita Castañeda***, A. Castro Luna****, J. Eleodoro Villavicencio Gastelú *****

\section{RESUMEN}

La diabetes mellitus tipo 2 es una enfermedad metabólica que en estos últimos años viene creciendo en varias regiones del mundo, siendo considerada una pandemia. Entre las complicaciones que se presenta en esta patología, se menciona a la periodontitis como la sexta complicación más común. Así también la diabetes es el mayor factor de riesgo para desarrollar periodontitis, incrementándose tres veces la posibilidad de periodontitis en estas personas, mencionándose el concepto de bidireccionalidad del complejo diabetes-periodontitis. En el presente caso clínico se plantea la posibilidad de uso como apoyo a la terapia periodontal de un producto natural obtenido de la amazonia peruana que por su propiedad antibacteriana estudiada, así como su fácil manejo y costo accesible sería una posible opción para su uso como coadyuvante en la terapia periodontal no quirúrgica en pacientes diabéticos tipo 2 con periodontitis

PALABRAS CLAVE: Diabetes mellitus tipo 2; Periodontitis; Productos naturales; Tratamiento.

\section{ABSTRACT}

Diabetes mellitus type 2 is a metabolic disease that in recent years has been growing in several regions of the world, being considered a pandemic. Among the complications that occur in this pathology, periodontitis is mentioned as the sixth most common complication. Diabetes is also the greatest risk factor for developing periodontitis, increasing the possibility of periodontitis three times in these people, mentioning the concept of bidirectionality of the diabetes-periodontitis complex. In the present clinical case, the possibility of use as a support to the periodontal therapy of a natural product obtained from the Peruvian Amazon, which because of its antibacterial property studied, as well as its easy handling and accessible cost, would be a possible option for its use as an adjunct. in non-surgical periodontal therapy in type 2 diabetic patients with periodontitis.

* $\quad$ Facultad de Odontología Universidad Nacional Mayor de San Marcos, Lima, Perú.Doctor en Estomatología. Magister en Microbiología. Cirujano Dentista.

* $\quad$ Facultad de Odontología Universidad Nacional Mayor de San Marcos, Lima, Perú. Doctor en Odontología. Cirujano Dentista.

*** Facultad de Odontología Universidad Nacional Mayor de San Marcos, Lima, Perú. Magister en Docencia e Investigación en Estomatología. Cirujano Dentista.

**** Facultad de Farmacia y Bioquímica Universidad Nacional Mayor de San Marcos, Lima, Perú. Doctor en Farmacia y Bioquímica.

***** Facultad de Odontología Universidad Nacional Mayor de San Marcos, Lima, Perú. Magister en Estomatología. Cirujano Dentista. 
KEY WORDS: Diabetes mellitus type 2; Periodontitis; Natural products; Treatment.

Fecha de recepción: 20 de octubre de 2019

Fecha de aceptación: 10 de noviembre de 2019

D. Ramos-Perfecto, L. Maita Véliz, L. Mariano Maita Castañeda, A. Castro Luna, J. Eleodoro Villavicencio Gastelú. Periodontitis en la diabetes tipo 2 y la copaiba como coadyuvante del tratamiento: Reporte de casos. $2021 ; 37,(2): 60-66$

\section{INTRODUCCIÓN}

La diabetes mellitus es una enfermedad no infecciosa que va creciendo, el número de pacientes diagnósticos a nivel mundial. Según la Organización Mundial de la Salud (OMS), se estima que hay 387 millones de personas con esta patología, siendo aproximadamente 25 millones identificados en la región sudamericana y el caribe, estimándose que para el 2030 sea la séptima causa de muerte ${ }^{(1)}$.

En el Perú presenta una prevalencia de 6,1 a 7\%. En la región costa es de $8,2 \%$, en la sierra $4,5 \%$ y en la selva 3,5\%. De todos los casos la diabetes tipo 2 representa el 96,8\%, estando relacionada a la obesidad del paciente, así como a una vida muy sedentaria ${ }^{(1-3)}$.

La diabetes es considerada una enfermedad con gran connotación desde fines del siglo XX y comienzos del XXI, aunque sus características clínicas son registrados en los papiros de Ebers (1550 A.C.) ${ }^{(4)}$, es en la actualidad una enfermedad que si no es controlada, puede afectar órganos tan importantes como el de la visión, riñón, sistema nervioso periférico, sistema cardiovascular, así como el estado periodontal de la pieza dentaria ${ }^{(5)}$.

La sintomatología más resaltante en el paciente diabético es la polidipsia, poliuria y disminución de peso de forma inexplicable, acompañándose de otros como la polifagia y la visión borrosa. Esta sintomatología se acompaña con niveles de glucosa en plasma de $\geq 200 \mathrm{mg} / \mathrm{dL}$ en algún momento del día, independientemente del tiempo transcurrido de la última comida o medidas de glucosa plasmática preprandial $\geq 126 \mathrm{mg} / \mathrm{dL}$, identifican la condición de diabetes ${ }^{(5,6)}$.

En la fisiopatología de la enfermedad influye mucho la presencia de los productos finales de glucosilación (AGE), estos afectan a las células endoteliales y monocitos, haciéndolas más susceptibles a los estímulos, que inducen a las células a producir mediadores inflamatorios. El acumulo de AGE a ni- vel de los tejidos y plasma está relacionado con las distintas complicaciones presentes en la persona diabética ${ }^{(5,7)}$.

Actualmente se viene manejando el concepto de la bidireccionalidad en la relación diabetes-periodontitis, los estudios actuales concluyen que la periodontitis, es la sexta complicación de la diabetes, generando con la cronicidad y el mal control de la enfermedad, pérdida de la estructura dental ${ }^{(7,8)}$ y que la susceptibilidad a desarrollar periodontitis es incrementada tres veces en la persona con diabetes (9). Estudios en pacientes diabéticos no controlados hispanos latinos informan que tienen la posibilidad de pérdida de nueve a más dientes, principalmente por periodontitis, indicando que los odontólogos deben poner mayor atención al atender pacientes hispanos latinos, evaluando su control metabólico de glucosa, solicitando pruebas que midan el nivel de glucosa en sangre, encontrando un diagnóstico temprano de la diabetes, lo que generaría, en el mejor de los casos un tratamiento temprano de la enfermedad evitando a futuro las complicaciones más severas de la diabetes ${ }^{(10)}$.

En relación al tratamiento de la periodontitis que se debe dar al paciente diabético tipo 2, en la actualidad y según evidencia, el tratamiento no quirúrgico periodontal es el más indicado y puede ser acompañado con un producto de apoyo sea este un antimicrobiano, antibiótico o producto natural con principios activos, que apoyen el tratamiento periodontal.

La oleorresina de copaiba es obtenida por perforación de un árbol que puede llegar a medir hasta 40 metros, su uso como cicatrizante, antimicrobiano y otras propiedades son utilizadas ancestralmente por pobladores de diferentes regiones del mundo, para tratar sus enfermedades ${ }^{(11)}$. En el Perú con sus tres regiones nos brindan una variedad de plantas con propiedades antibacteriana, cicatrizante, siendo una de ellas la copaiba. Este producto está conformado por sesquiterpenes y diterpenes, como: ß-cariofi- 
leno, $\beta$-elemeno, $\beta$-selineno, $\boldsymbol{\alpha}$-cariofileno, etc. Su propiedad antibacteriana así como nula toxicidad ante un uso adecuado, su fácil manejo y transporte hacia el surco gingival, lo hacen un producto de posible uso en la periodontitis ${ }^{(11-16)}$.

En el caso clínico presentado, se tiene como objetivo proponer el uso de la copaiba como posible apoyo al tratamiento periodontal no quirúrgico de la periodontitis en diabéticos tipo 2 .

\section{DESCRIPCIÓN DE CASOS CLINICOS}

\section{CAso 1}

Paciente identificado con las iniciales CMM, con 65 años de edad, de sexo masculino, es examinado como parte del despistaje de periodontitis en pacientes diabéticos en un hospital de referencia. Es diagnosticado de diabetes tipo 2, hace 3 años, recibiendo tratamiento a base de metformina mañana y noche y glibenclamida después del almuerzo. Aparentemente es un paciente es un estado de salud estable con registro de hemoglobina glicosilada al iniciar el tratamiento periodontal de 6,8 \%.

En sus antecedentes médicos generales menciona que ha sufrido de afecciones a nivel prostático, por el cual, recibió tratamiento.

Entre sus antecedentes odontológicos, refiere que hace 40 años se realizó un tratamiento de ortodoncia y la última vez que acudió al odontólogo fue hace tres años para realizarse una exodoncia. En relación al cuidado de su dentadura, refiere que su higiene es diaria, cepillándose los dientes una sola vez al día. Ha observado sangrado de sus encías y ha perdido dientes con movilidad y considera que tiene un mal estado de la salud de su boca. Así también al control de placa que se realizó, presento un índice de higiene oral de O’Leary del 92\%. En el momento del examen, el paciente no recibía tratamiento periodontal y no tenía medicación antibiótica ni utilizada algún agente antimicrobiano como parte de su higiene bucal.

Previo al tratamiento y recibiendo la información pertinente del ensayo clínico que se va a realizar en su persona como parte del tratamiento a recibir, el paciente firmo el documento de consentimiento informado (con acta de evaluación ética $\mathrm{N}^{\circ} 0288$ y código de proyecto $\mathrm{N}^{\circ} 0368$ del comité de ética de la Facultad de Medicina de la Universidad Nacional mayor de

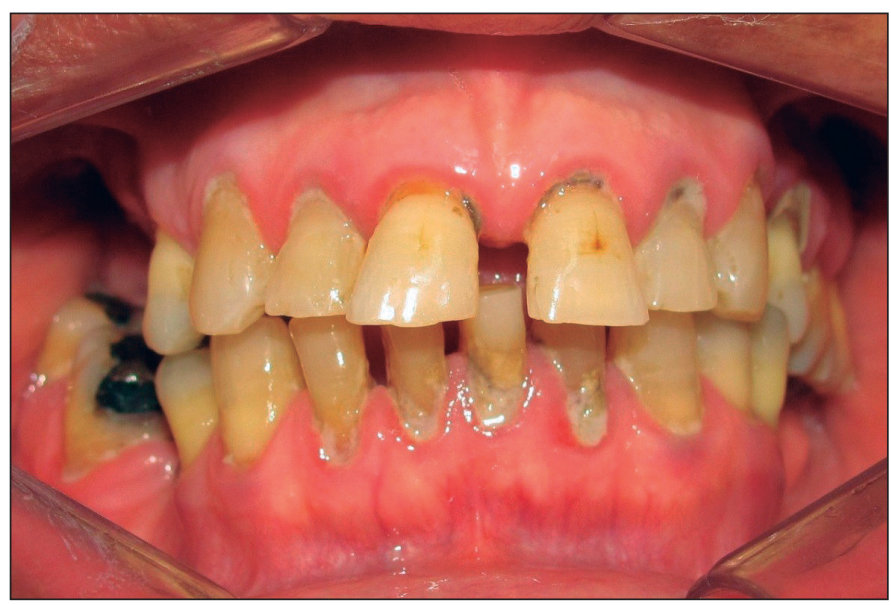

Figura 1. Paciente diabético tipo 2 con periodontitis, en la pza. 11 y 21, previo al inicio del tratamiento periodontal no quirúrgico.

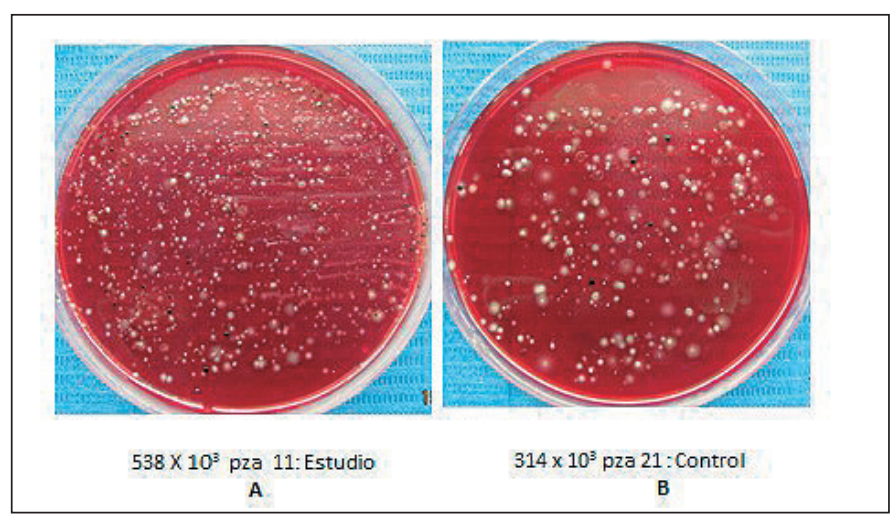

Figura 2. Recuento Microbiano antes del desarrollo del tratamiento no quirúrgico periodontal, se detecta una variedad de colonias confirmando la naturaleza polimicrobiana de la periodontitis. Zona de estudio pieza 11 (A), Zona de control pieza 21 (B).

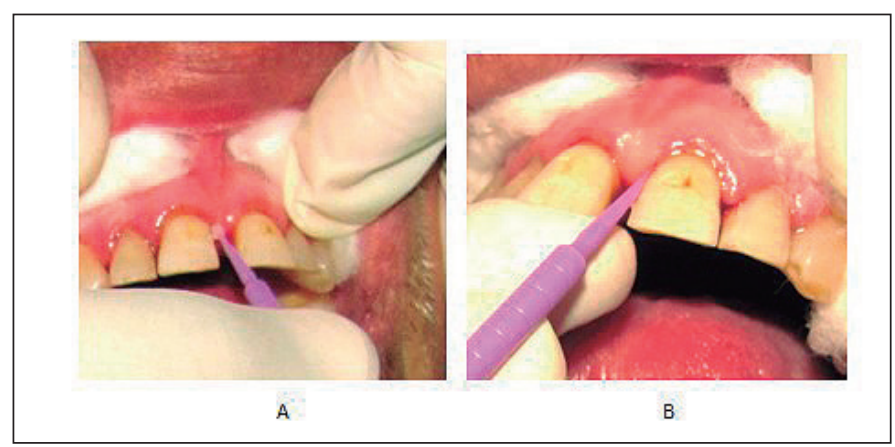

Figura 3. Cargados los microcepillos con $10 \mu \mathrm{L}$, se lleva el producto natural a la bolsa periodontal de la pza. 11 (A) y la clorhexidina a la bolsa periodontal de la pza. 21 (B).

San Marcos), detallándole, las fechas de su participación, las tomas de muestras y los estudios a realizar.

Comentando que todo los procedimientos son autofinanciado por el investigador y que el paciente es libre 
de retirarse en cualquier momento de la investigación.

\section{Diagnóstico}

Luego de un estudio periodontal registrado en un periodontograma con los siguientes ítems: profundidad al sondaje, sangrado al sondaje, nivel de margen de la encía, nivel de inserción clínica y presencia de placa. El diagnóstico periodontal del paciente fue de una Periodontitis moderada. Se planteó como tratamiento, la terapia periodontal no quirúrgica y la aplicación como apoyo al tratamiento, de copaiba en un área de estudio específica, y en el área homologa de control a la clorhexidina al 0,12\%, para evaluar cambios en el trascurso de la evolución del tratamiento.

\section{Plan de Tratamiento}

Se confirmó el diagnóstico y condición del paciente, firmando él paciente a libre voluntad el documento de consentimiento informado. Se realizó una historia periodontal con su respectivo periodontograma, identificando las piezas dentarias homologas con periodontitis, se registró como parte del diagnóstico, la medición de sus bolsas periodontales, el nivel de inserción clínica, sangrado y color de encía. Este procedimiento se llevara al inicio, como a los dos meses después del tratamiento. Se tomó muestras de la placa subgingival del área diagnosticada con periodontitis, con la finalidad de hacer un recuento de las unidades formadoras de colonias (UFC). Asî como una muestra de sangre para evaluar el control de su nivel de glucosa en sangre y determinar su hemoglobina glicosilada (Hb1Ac).

Se ejecuta la fase I de la terapia periodontal no quirúrgica, que consiste principalmente en retirar placa supra e subgingival y tártaro de la bolsa periodontal, por medios mecánicos con instrumentos rígidos, curetas (Gracey, EEUU) y raspadores (Gracey, EEUU). La aplicación de copaiba previamente esterilizado, recolectada de la amazonia del Perú, así como del producto control clorhexidina al 0,12 \% (Dentodex, Intralab) fueron aplicados por un profesional colaborador, que desconoce los productos y que fue informado de la forma de aplicación en la zona a evaluar. Esto se realizó una vez finalizado la fase I del tratamiento periodontal. La aplicación fue por medio de un microbrush (micro-applicators, China) de fibras no absorbentes, con parte activa de $1,5 \mathrm{~mm}$ de diámetro de color lavanda tenue, cargada con10 $\mu \mathrm{L}$ de copaiba que recorrió el surco gingival patológico,

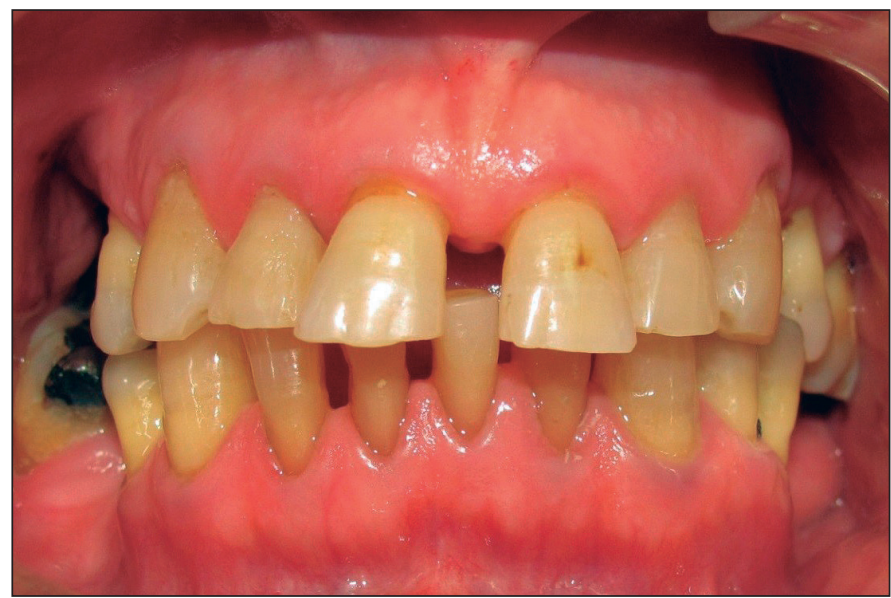

Figura 4. Aspectos clínicos 2 meses después del tratamiento periodontal no quirúrgico y la aplicación de los productos coadyuvantes.

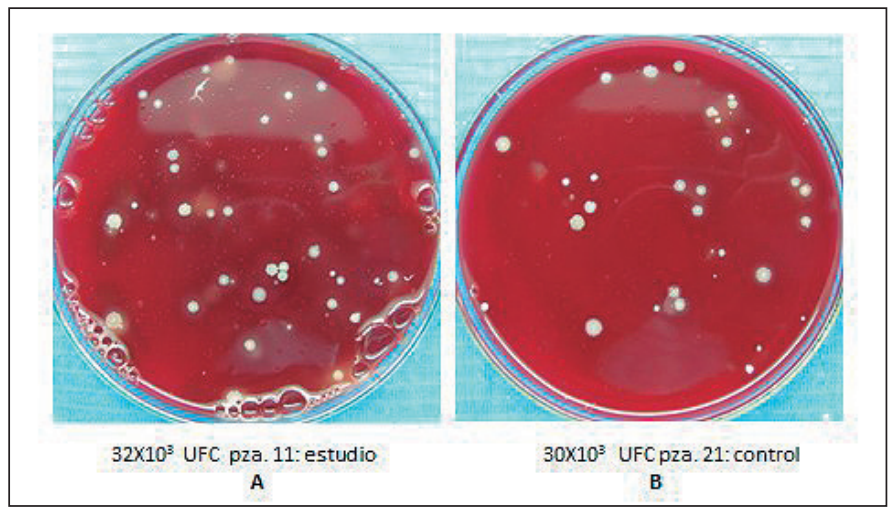

Figura 5. Recuento Microbiano después del tratamiento periodontal no quirúrgico y aplicación de coadyuvantes. Se detecta una disminución de UFC en ambas zonas estudiadas, ausencia de colonias negro pigmentadas. La mayor disminución de microorganismos se obtuvo en la zona de estudio (A), resultados similares se obtuvieron en la zona de control (B).

zona diagnosticada con periodontitis (zona de estudio) y en la zona homologa relacionada a la pieza dentaria, se aplicó con otro igual instrumento $10 \mu \mathrm{L}$ de clorhexidina al 0,12 \% (zona control). Previa a la aplicación de los productos, las zonas debieron recibir un aislamiento relativo con torundas de algodón. Las zonas aplicadas con los productos deben mantenerse sin contacto de saliva o la mucosa del labio por un período de 60 segundos.

La evaluación y mantenimiento del tratamiento se realizó en la primera, segunda, tercera, cuarta semana y a los dos meses, después del tratamiento. En cada evaluación se aplicaran los productos según sean zona de estudio o de control. Así también en la última fecha de evaluación se tomaron la segunda muestra de placa subgingival para un recuento de bacterias y una muestra de sangre, para el control de su Hb1Ac. 
La última evaluación a los dos meses, se realizó un segundo periodontograma evaluando los cambios a nivel del surco gingival y observar la ganancia del nivel de inserción.

\section{RESULTADOS}

Luego de un período del tratamiento periodontal no quirúrgico, de dos meses, se aprecia que las zonas de estudio y de control, presentaron una gran mejoría, encontrando resultados similares en ambos grupos de estudio, las bolsa periodontales se reducen en el caso de la zona de estudio con el producto natural de 4 a $2 \mathrm{~mm}$ y en caso de la zona control con clorhexidina de 5 a $2 \mathrm{~mm}$. También se observa cambio de color de la encía a un rosado coral clásico, hay ganancia de inserción por disminución de surcos y ausencia de sangrado al sondaje.

En relación a los parámetros microbiológicos ambos lograron reducir sus recuentos microbianos, presentado, disminuciones en la zona de estudio de $538 \times 10^{3}$ a $32 \times 10^{3}$ y en la zona de control de $314 \times 10^{3}$ a $30 \times 10^{3}$. Enfatizando que la mayor reducción de unidades formadoras de colonias (UFC) se presentó en la zona de estudio en la que se usó el producto natural, esta disminuyo en $506 \times 10^{3}$ UFC, lo que indicaría una disminución considerable de la carga microbiana en el surco gingival de la zona de estudio.

En relación a la prueba que indica el control metabólico de la glucosa, la $\mathrm{Hb} 1 \mathrm{AC}$, se reduce de $6,8 \%$ a $5,4 \%$, lo cual indica el buen control de la glucosa en el paciente diabético tipo 2 con periodontitis

\section{Caso 2}

Paciente diabético de tipo 2 de 53 años, con diagnóstico de periodontitis moderada a nivel de la pieza dentaria 31. El paciente tiene un tratamiento de la diabetes a base de metformina de toma diaria.

Una vez obtenido el diagnóstico el plan de tratamiento a seguir es similar al realizado en el caso 1 .

\section{DISCUSIÓN}

La terapia periodontal no quirúrgica es considerado como el "gold estándar" de los procedimiento para el tratamiento de la periodontitis ${ }^{(17,18)}$, más aún en

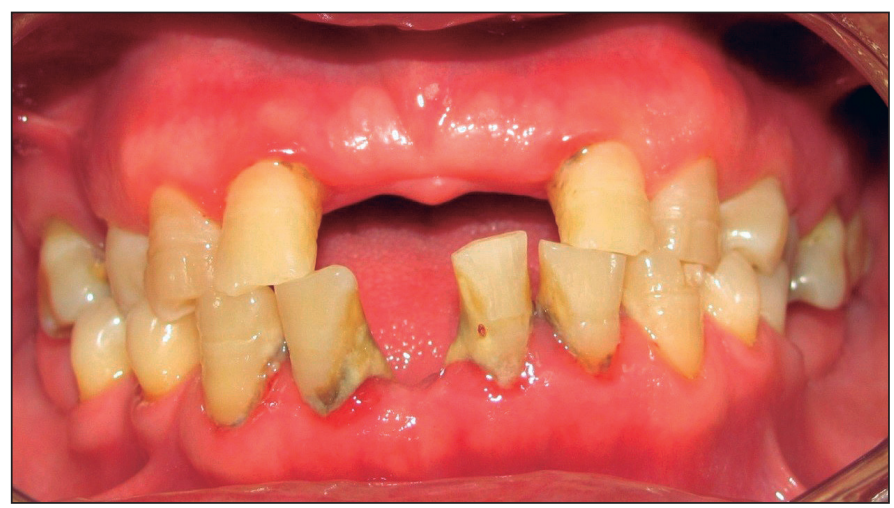

Figura 6. Condición en la que se diagnostica, la periodontitis en la cavidad bucal del paciente, obsérvese la acumulación de placa denta, así también los cambios de volumen y color de la encía.

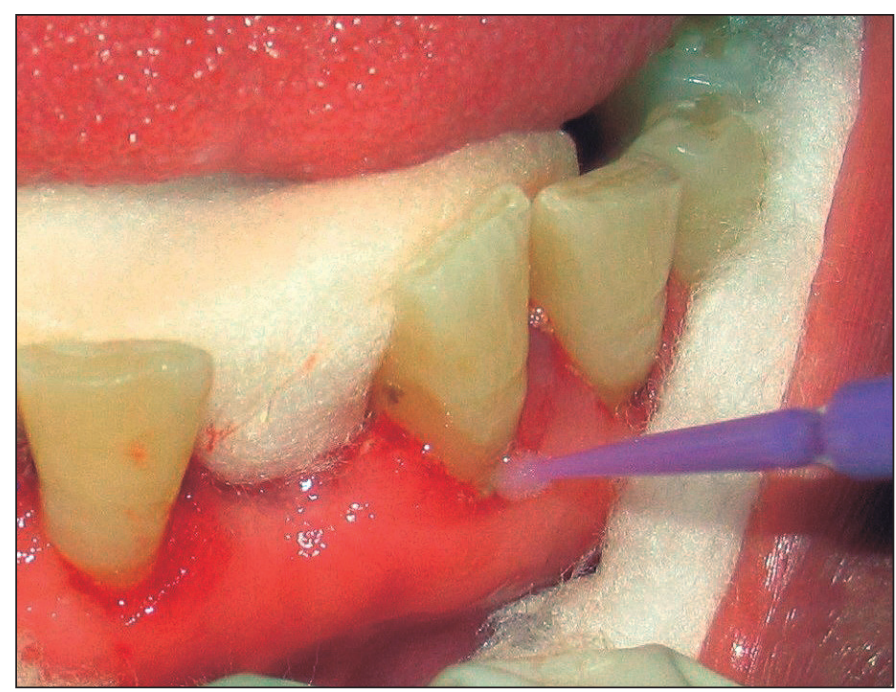

Figura 7. Luego del retiro de la placa subgingival y supragingival se aplica $10 \mu \mathrm{L}$ de copaiba a nivel subgingival con un dispositivo que permite su ingreso a profundidad, obsérvese el aislamiento relativo, para evitar el ingreso de saliva, tiempo de contacto directo de la copaiba, 60 segundos.

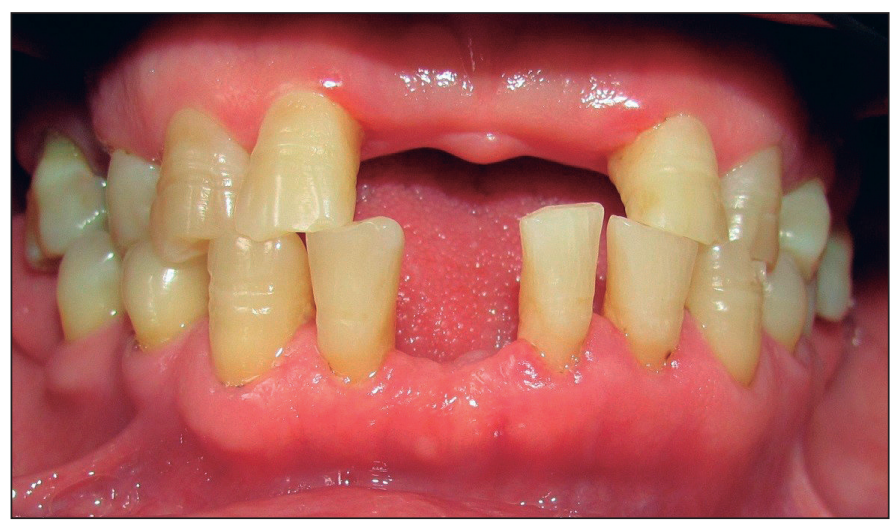

Figura 8. A los dos meses Obsérvese la condición saludable de la encía queratinizada de la pieza 31 , ausencia del color eritematoso, así como una condición de salud periodontal, sumado a ello el buen control de placa dental. 
pacientes diabéticos tipo 2 que cursan una patología sistémica. La presencia localizada o generalizada de la periodontitis agrava la condición sistémica. Mauri et $a l^{(19)}$ y Sanz et ${ }^{(20)}$ apoyan a la terapia periodontal no quirúrgica (TPNQ) como tratamiento de la periodontitis en pacientes diabético tipo 2, con mejoras en los parámetros más importantes: reducción en la profundidad al sondaje, ganancia del nivel de inserción clínica, así como otros parámetros. En el caso presentado, la TPNQ demuestra resultados positivos mejorando los parámetros de profundidad al sondaje (PS), nivel de inserción clínica (NIC), sangrado al sondaje (SS), cambios de la coloración de la encía principalmente.

El procedimiento realizado en el caso clínico fue básicamente la fase I de la terapia periodontal, realizando el raspaje y alisado radicular, acompañado de una motivación para el cuidado de su higiene dental, mejoras en su fisioterapia y como apoyo antibacteriano se aplicó un producto natural, en la zona de estudio y en la zona control se utilizó la clorhexidina "gold estándar" de los antimicrobianos utilizados en patologías periodontales. Los resultados en ambos casos logran mejorar los parámetros periodontales mencionados anteriormente. Aunque la literatura actual no tiene nada concluyente de los beneficios de los antimicrobianos como apoyo a la TPNQ, investigadores como Rovai et $\mathrm{al}^{(21)}$ indican que el uso de antimicrobianos de uso tópico local en el apoyo de la TPNQ lograron reducir significativamente los parámetros de PS, NIC en pacientes diabéticos tipo 2, en bolsas periodontales mayores de $5 \mathrm{~mm}$. Así también Duarte et $a l^{(22)}$ en la línea contraria, menciona que la TPNQ acompañada con antibióticos (amoxicilina y metronidazol) en pacientes diabéticos tipo 2 con periodontitis y pacientes con periodontitis sin diabetes luego de un monitoreo de 12 meses, encontró que los pacientes no diabético con periodontitis severa no responden mejor que los pacientes diabéticos tipo 2 al tratamiento realizado. Una posición más moderada es la que tiene Sanz et $a l^{(20)}$ quien manifiesta que no son concluyentes los estudios que manifiestan que los antimicrobianos como apoyo a la TPNQ generen un beneficio mayor al solo procedimiento de la TPNQ.

En relación a la capacidad antibacteriana del producto natural, estudios de varios investigadores sustentan sus beneficios contra una diversidad de microorganismos como Porphyromanas gingivalis y Streptococcus mutans, así también como a microorganismos asociados a patologías sisté- micas. Su aplicación de fácil ejecución como la innovación de aplicar microcepillos, hacen que llegue el producto a profundidad en la bolsa periodontal a una dosis adecuada, generando una mayor reducción de las UFC comparándolo con la zona de control de la pieza homologa con periodontitis, del paciente diabético tipo 2, haciendo mención que la zona control utiliza como apoyo a la clorhexidina. Estudios clínicos han evaluado el uso de antibióticos como apoyo a la TPNQ, identificando cambios microbiológicos. Tamashiro et $a l^{(23)}$ evaluando la flora subgingival en pacientes diabéticos que recibieron TPNQ más antibióticos, encontraron que este grupo de estudio presento proporciones bajas de patógenos del complejo rojo (Porphyromonas gingivalis, Tannerella forsythia, Treponema denticola) alcanzando un $5,5 \%$, y el grupo control $12,5 \%$, concluyendo que la TPNQ mejora las condiciones microbiológicas. El estudio realizado por Tsobgny et al. ${ }^{(24)}$ que utiliza como apoyo a la clorexidina a la TPNQ de pacientes diabéticos tipo 2 con periodontitis, por un período de tres meses de estudio, pudo determinar que se reducen los parámetros clínicos periodontales de la enfermedad periodontal. También se logró reducir la $\mathrm{Hb} 1 \mathrm{AC}$ en estos pacientes en un 2,2\%. Si bien los beneficios periodontales que se lograron en el caso clínico son importantes, el nivel de la $\mathrm{Hb} 1 \mathrm{AC}$, también disminuye de $6,8 \%$ a $5,4 \%$, indicando el buen control metabólico de la glucosa en el paciente diabético tipo 2. Para completar la información en relación a la $\mathrm{Hb} 1 \mathrm{AC}$, los estudios que evalúan los beneficios de la TPNQ en el paciente diabético tipo 2 con periodontitis, manifiestan que hay reducción significativa de los valores de esta prueba bioquímica, así lo comprobaron Botero et al. ${ }^{(25)}$, Palka et al. ${ }^{(26)}$, Preshaw et al. ${ }^{(9)}$. Los resultados de este estudio son similares a los mencionados anteriormente.

\section{CONCLUSIÓN}

De lo realizado en los casos clínicos presentados se puede dar indicios de los beneficios de la copaiba como apoyo a la TPNQ en el tratamiento de la periodontitis de pacientes diabéticos tipo 2, aunque más estudios con una muestra mayor sería necesario realizar para concluir su uso.

Los autores agradecen a la Universidad Nacional Mayor de San Marcos, por el apoyo en el desarrollo de la investigación. 


\section{REFERENCIAS BIBLIOGRÁFICAS}

1. Villena JE. Epidemiologia de la diabetes mellitus en el Perú, Diagnóstico 2016:55(4):173-81.

2. Ardila E. Perimetro de cintura aumentada y riesgo de diabetes. ActaMedicColomb. 2016;41(3):161-2.

3. Pham T, Tran T. The interaction among obesity, type 2 diabetes mellitus, and periodontitis in Vietnamese patients. ClinExp Dent Res. 2018;4(3):63-71.

4. Sanchez RG. Historia de la diabetes. Gac Med Bol. 2007;30(2):74-8.

5. Shamim L Ahmad. Diabetes: and old disease, a new insight, 1ra ed. USA: Lander Bioscience.2012:76-87.

6. ADA. Diagnosis and classification of diabetes mellitus. Diabtes Care. 2007;30(1):542-7.

7. Bascones-Martinez A, Muñoz-Corcuera M, Bascones-Ilundaín J. Diabetes y periodontitis: una relación bidireccional. Medcli. 2015;145(1):31-5.

8. Ziukaite L, Slot D, Van der weijden F. Prevalence of diabetes mellitus in people clinically diagnosed with periodontitis: A systematic review and meta-analysis of epidemiologic studies. $\mathrm{J}$ ClinPeriodontol. 2018;45(6):658-62.

9. Presshaw P, Alba L, Herrera D, Jepsen S, Konstantinidis A, Makrilakis K, et al. Diabetologia 2012;55:21-31.

10. Greenblatt A, Salazar Ch, Northridge, Kaplan R, Taylor G, Finlayson T, Qi Q, et al. Association of diabetes with tooth loss in Hispanic/Latino adults: findings from the Hispanic community health study/ Study of latinos. BMJ Open Diab Res Care 2016;4:e000211.doi:10.1136/bmjdrc-2016-000211

11. Casariego ZJ. Mecanismo de acción de plantas medicinales aplicadas en lesionesestomatológicas: revisión. Av Odontoestomatol. 2016;32(1):35-44

12. Pieri FA, Messi MC, Moreira MAS. Histórico, extração, aplicações industrias e propriedadesmedicinais. RevBras PLMedBotucatu. 2009;11(4):465-72.

13. Leandro LD, Souza VF, Souza BPC, Oliveira NJK, Da Silva JA, Veiga JVF. Chemistry and Biological activities of terpenoids from Copaiba (Copaiferaspp) oleoresins. Molecules 2012;17(4):3866-89.

14. Sachetti CG, Carvalho RR, Paumgartten FJ, Lameira OA, Caldas ED. Developmental toxicity of copaiba tree (Copaifera reticulate Ducke, Fabaceae) oleoresin in rat. Food ChemToxicol. 2011;49:1080-5.

15. Ramos PD, Castro CA. Actividad antibacteriana de Copaiferareticulata "copaiba" sobre Porphyromonasgingivalis aisladas de pacientes con periodontitis. Odontol Sanmarquina. 2014;17(1):7-11.

16. Diefenbach A, Gomez M, Rivera H, Kuchenbecker C. Antimicrobial activity of copaiba oil (Copaifera ssp.) on oral pathogens: Systematic review. Phytother Res. 2018;32(4):586-96

17. Teles RP, Haffajee AD, Socransky SS. Microbiological goals of periodontal therapy. Periodontol 2000. 2006;42:180---218.

18. Maita VL, Ramos PD, Castañeda MM, Galvez CL, Maita CL, Navarro CC. Presencia de bacterias negro pigmentadas en la pared de la bolsa periodontal al inicio y ter- mino de la terapia periodontal. Odonto Sanmarquina. 2016;19(1):47-51.

19. Mauri E, Merlos A, Estrugo A, Jane E, Lopez LJ, Viñas $M$. Benefits of non-surgical periodontal treatment in patients with type 2 diabetes mellitus and chronic periodontitis : A randomized controlled trial. J Clin Periodontol. 2018;45(3):345-53

20. Sanz M, Ceriello A, Buysschaert M, Chapple I, Demmer R, Graziani $F$, et al. Scientific evidence on the links between and guidelines of the joint workshop on periodontal diseases and diabetes by the international diabetes federation and the European Federation of Periodontology. J Clin Periodontol. 2018;45:138-49.

21. Rovai ES, Souto ML, Ganhito JA, Holzhausen M, Chambrone L, Pannuti CM. Efficacy of local antimicrobials in the non-surgical treatment of patients with periodontitis and diabetes : A systematic Review. J Periodontol. 2016;87(12):1406-17.

22. Duarte PM, Feres M, Yassine LLS, Soares GMS, Miranda TS, Faveri $M$, etal.Clinical and microbiological effects of scaling and root planning, metronidazole and amoxilin in the treatment subjects with periodontitis: a cohort study. J Clin Periodontol. 2018;45(11):1326-35.

23. Tamashiro N, Duarte P, Miranda T, Maciel S, Figueiredo L, Faveri M, et al. J Dent Res. 2016;95(7):829-36.

24. Tsobgny N, Lontchi E, Nana, A, Tankeu A, Katte J, Mesmin $Y$, et al. Effects of nonsurgical periodontal treatment on glycatedhaemoglobin on type diabetes patients (PARODIA 1 study): a randomized controlled trial in a sub-saharan Africa population. BMC Oral Health. 2018;18(1):28-35.

25. Botero JE, Rodriguez C, Agudelo A. Periodontal treatment and glycaemic control in patients with diabetes and periodontitis: an umbrela review. Aust Dent J. 2016;61:134-48.

26. Palka K, Narula S, Rajput R, Sharma R, Tewari Sh. Periodontal and glycemic effects of nonsurgical periodontal therapy in patients with type diabetes stratifield by baseline HB1AC. J Oral Sci. 2015;57(3):201-11.

\section{CORRESPONDENCIA:}

Donald Ramos Perfecto

Correo electrónico: dramosp@unmsm.edu.pe

Av. German Amezaga 375, ciudad universitaria

UNMSM, Lima, Perú

Coautor:

Luis Maita Véliz

luismv21@yahoo.com

Luis Mariano Maita Castañeda

lmaitac@unmsm.edu.pe

Américo Castro Luna

caslasha@hotmail.com

Jorge Eleodoro Villavicencio Gastelú

jvillavicenciog@unmsm.edu.pe

Conflicto de interés: los autores declaran no tener conflictos de interés.

Fuente de financiamiento: autofinanciado

El manuscrito enviado no se encuentra en estos momentos bajo ninguna revisión editorial en alguna otra revista científica. 